UK. Observation studies of OPM suggest substantially lower mortality than IPM data in National HF audits. This suggests observation bias.Aims: To determine: (i) if there has been expansion in OPM service in UK centres; (ii) what proportion of patients are treated with OPM; (iii) what proportion of HF service has clinical psychology support; and (iv) feasibility of conducting a multicentre randomised controlled trial (RCT) in the UK to test safety and cost-effectiveness of OPM. Methods and

Results The 'AHF-IN or OUT-Survey' was sent using Survey Monkey software via the UK HF Investigators Research Network and local Clinical Research Network to hospitals in the UK in 2021.53 responded and estimated 25485 patients with AHF receive inpatient care per year-median 600 per site [IQR 295,800]: 2730 patients \{median 50 [7,100] per site\} are treated per year with OPM, representing $9.7 \%$ of the total population of patients who had AHF. 67\% (35 of 53) centres provided access to OPM. Of the 17 which do not (33\%), 11 of these hope to develop this service while 4 do not know if they should develop this service. Two sites had no intention to develop this service. 4 provide Subcutaneous (SC) diuretics only whilst the majority (31) provide IV diuretic service, including 9 which can deliver both IV and SC

\begin{tabular}{lcc}
$\begin{array}{l}\text { Abstract } \mathbf{1 3 3} \text { Table } \mathbf{1} \\
\text { diuretic treatment? }\end{array}$ & Who administers outpatient based IV \\
\hline Frequency & $\%$ & 15.4 \\
\hline "Community IV team" & 8 & 5.7 \\
Ambulatory team & 3 & 1.9 \\
Cardiac outpatient nurses & 1 & 3.8 \\
District nurse & 2 & 32.7 \\
Heart failure specialist nurse & 17 & 1.9 \\
rapid response team & 1 & \\
( community ). & & \\
\hline
\end{tabular}

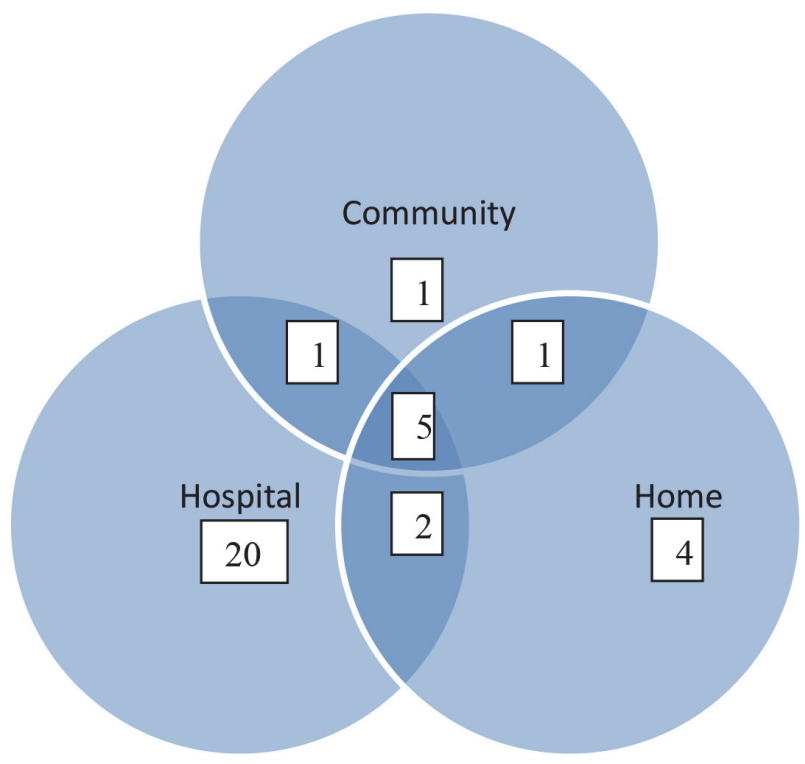

Abstract 133 Figure 1 Ways to provide outpatient based acute heart failure treatment
Diuretic Service. 28 provide Hospital based OPM ("frusemide lounge"/ambulatory care centre). There are 8 communitybased services and 12 sites can deliver parenteral diuretics at home (figure 2). Table 1 shows details of the healthcare professionals who administer outpatient based IV diuretic treatment.Only 19\% (10) have clinical psychology service; whilst $75 \%$ (39) do not. 6\% (3) of respondents are not sure if they have clinical psychology service. We are planning a multicentre RCT to test if indeed OPM of AHF is safe, cost-effective, improves wellbeing and hope. 3573 patients are eligible to take part in the SAFE-RCT over 2 years -median 64 per site $[33,100]$. 1501 can be randomised within 2 years \{median 20 per site $[30,50]\}$.

Conclusions Outpatient based IV diuretic therapy is gaining popularity in the UK. Only a small minority of hospitals have clinical psychology service for patients who suffer from HF. Before further rapid expansion of outpatient based IV diuretic service in the UK, a multicentre RCT is urgently needed to test the safety and cost-effectiveness of this innovative service. Importantly, there also needs to be expansion of clinical psychology service alongside in order to provide patients with excellent whole person care

Conflict of Interest No

\section{LANCASHIRE OBJECTIVE VOLUME EVALUATION OF LEG OEDEMA IN HEART FAILURE RANDOMIZED CONTROLLED CROSS-OVER TRIALS (LOVE-HF ARTIFICIAL INTELLIGENCE RESEARCH PROGRAMME): RATIONALE AND METHODOLOGY}

${ }^{1}$ Reham Awad, ${ }^{2}$ Robert Jones, ${ }^{2}$ Omar Assaf, ${ }^{1}$ Abdullah Abdullah, ${ }^{2}$ Alison Seed, ${ }^{2} \mathrm{CJ}$ Cassidy, ${ }^{1}$ Lesley Howard, ${ }^{2}$ Suzanne Wong, ${ }^{2}$ Rebecca Taylor, ${ }^{3}$ John Cleland, ${ }^{4}$ Deirdre Lane, ${ }^{5} \mathrm{G}$ Davis, ${ }^{3}$ Pierpaolo Pellicori, ${ }^{6}$ Rhiannon Berry, ${ }^{7}$ Kenneth Wong. ${ }^{1}$ Blackpool Teaching Hospitals NHS Foundation Trust (Blackpool Victoria Hospital), Blackpool, UK; ${ }^{2}$ Blackpool Victoria Hospital; ${ }^{3}$ University of Glasgow, UK; ${ }^{4}$ Liverpool Centre for Cardiovascular Science, University of Liverpool, UK; ${ }^{5}$ School of Medicine, University of Central Lancashire, Preston, UK; ${ }^{6}$ Heartfelt Technologies Ltd, Cambridge, UK; ${ }^{7}$ Blackpool Teaching Hospitals

\subsection{6/heartjnl-2021-BCS.131}

Introduction In England and Wales, heart failure (HF) was the primary diagnosis for $>81,000$ hospital admissions in 2016-17, with a 30-day readmission rate of nearly $20 \%$. The National HF Audit reports that half of these admissions were associated with moderate or severe peripheral oedema. Conventionally, leg oedema is assessed and graded by a healthcare professional (HCP). A serial measurement of weight is an alternative method of assessing water retention at home but this relies on patient compliance.The Heartfelt device uses high-resolution cameras to generate $3 \mathrm{D}$ images of the lower legs and calculates volumes with a precision of about $20 \mathrm{mLs}$ without the need for active patient input. Linking Artificial Intelligence algorithms may then be applied to inform either the patient or a healthcare professional of appropriate actions.

Methods The Lancashire Objective Volume Evaluation of leg oedema in Heart Failure (LOVE-HF) artificial intelligence research programme consists of two pilot randomised controlled, cross-over trials (each with 30 patients and 30 day assessment periods), a registry and screening log (the latter to permit comparison of baseline characteristics to ensure generalisability). 
Patients with HF who received IV diuretics (within $<6$ months) or with peripheral oedema despite receiving at least $80 \mathrm{mg} /$ day of oral furosemide (or equivalent), will be enrolled in LOVE-HF (comparing the Heartfelt device on top of standard care to standard care: figure 1) and LOVE-HF-2 (comparing the Heartfelt device to weight monitoring: figure 2). The efficacy outcome of interest is 'number of days alive out of hospital'. Secondary outcomes include serious adverse events, quality of life, mental wellbeing, hopelessness scores and HFsymptoms.

These vanguard trials will determine the feasibility of datacollection and confirm estimates of event rates that will inform the design of a substantial multi-centre trial (GLOVEHF- GLobal Objective Volume Evaluation of oedema in Heart Failure) to determine the clinical utility of the Heartfelt device.

\begin{tabular}{|l|l|}
\hline \multicolumn{2}{|c|}{ Pilot double blinded crossover interventional RCT } \\
\hline Pilot trial to compare Heartfelt device to standard care (LOVE-HF) \\
\hline & The primary end point is "days alive out of hospital". \\
\hline
\end{tabular}

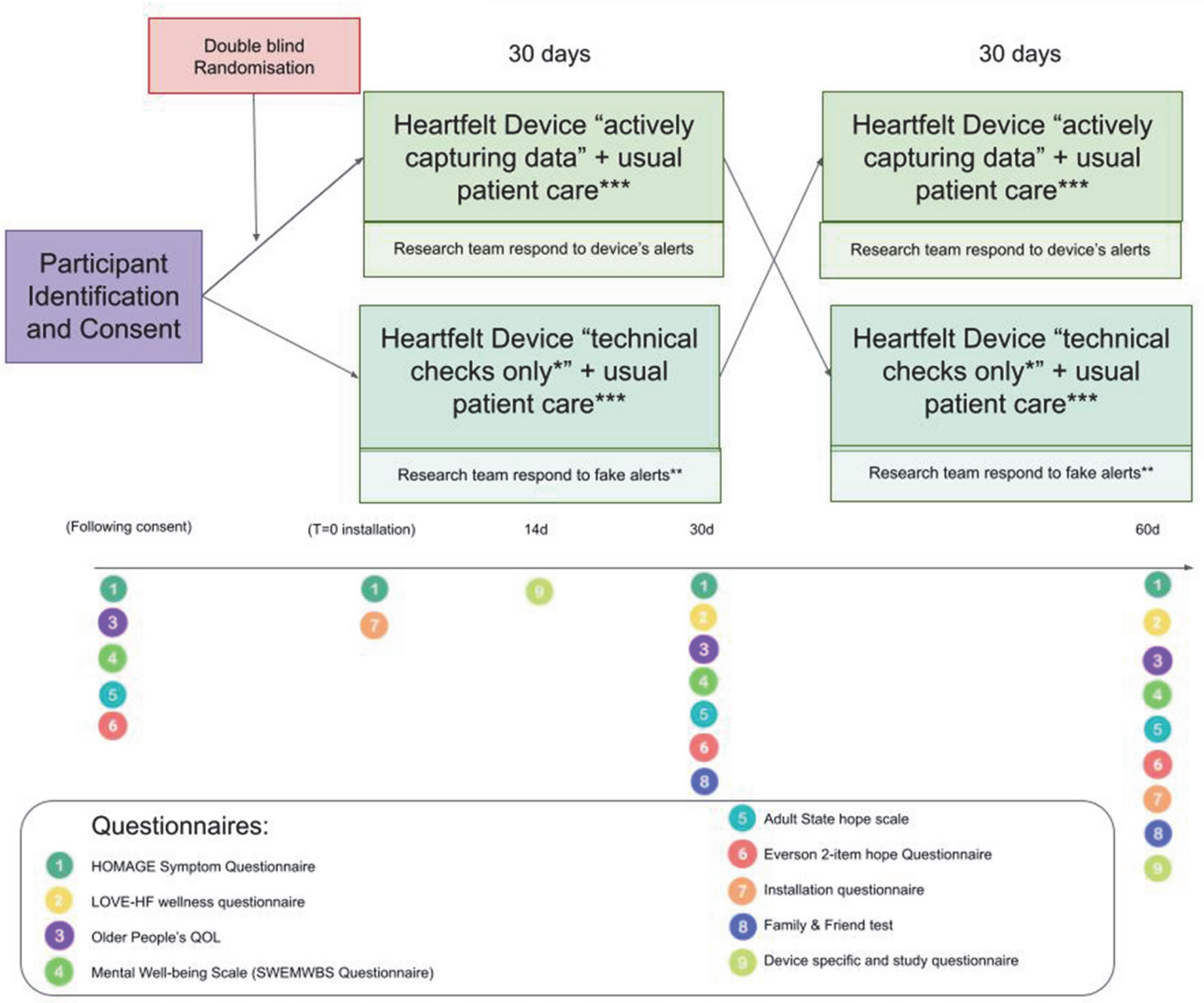

*In the "technical checks only" mode, the Heartfelt device will still capture data in the patient's home, but the volumes will not be calculated during the study. The volume calculations can be performed retrospectively, and used as part of the overall study analysis.

${ }^{* *} \mathrm{~A}$ fake alert are generated in the control group when a real alert is generated by the Heartfelt device in the active group.

$\star \star \star$ Standard care is defined as weighing if recommended by patient's HCPs with symptom reporting.

\section{Abstract 134 Figure 1}




\section{Pilot crossover interventional RCT}

Pilot trial to compare Heartfelt device to weighing scales (LOVE-HF2)

The primary end point is "days alive out of hospital".

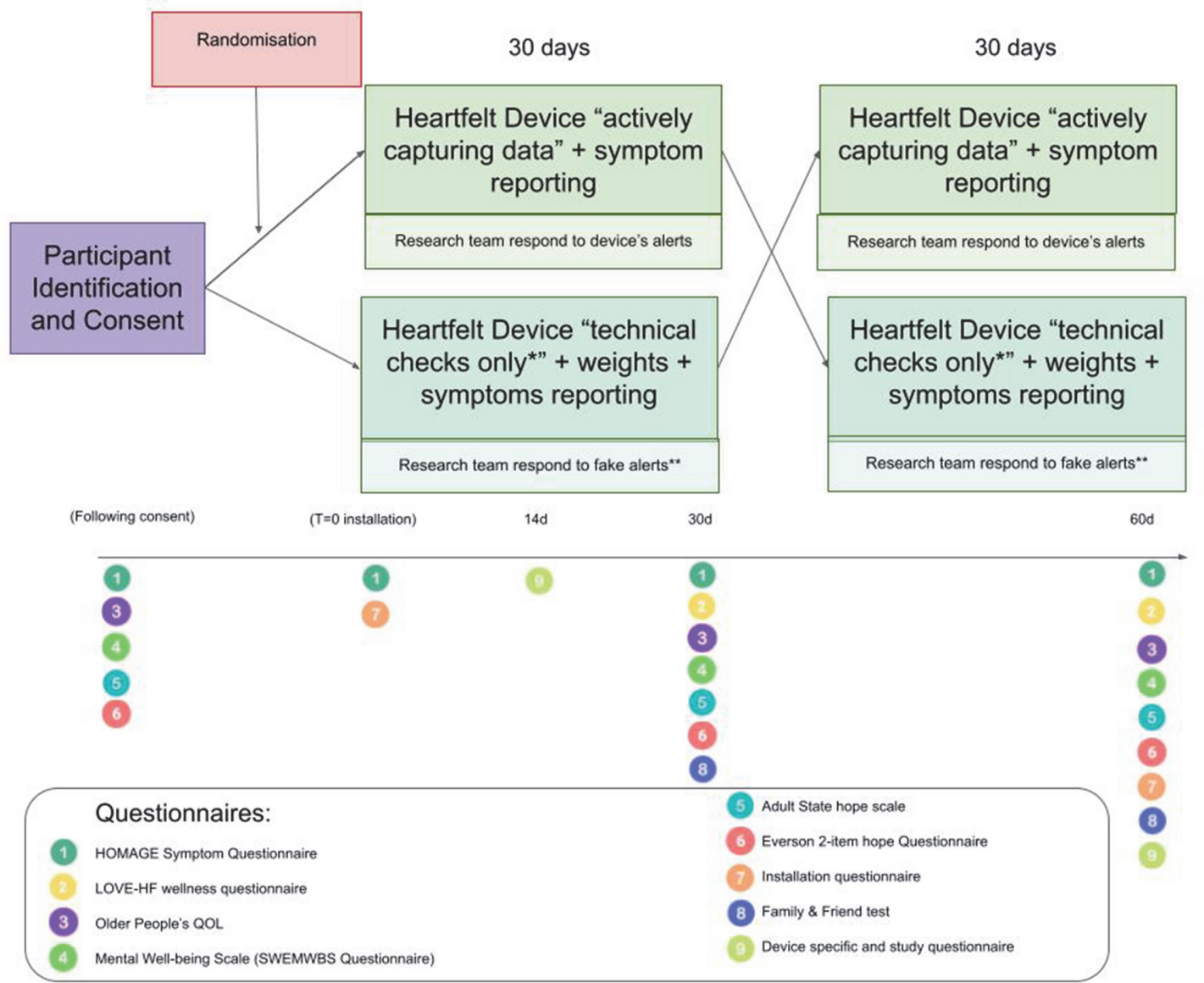

*In the "technical checks only" mode, the Heartfelt device will still capture data in the patient's home, but the volumes will not be calculated during the study. The volume calculations can be performed retrospectively, and used as part of the overall study analysis.

\section{Abstract 134 Figure 2}

Conflict of Interest Heart Failure

\begin{tabular}{l}
\hline 135 AN IMPROVED EJECTION FRACTION PARAMETER \\
CAPABLE OF REPRESENTING CARDIAC FUNCTION \\
REGARDLESS OF HEART MORPHOLOGY TO \\
DISTINGUISH HFPEF FROM NORMAL HEARTS
\end{tabular}

${ }^{1}$ Choon Hwai Yap, ${ }^{2}$ Yu Zheng, ${ }^{2}$ Wei Xuan Chan, ${ }^{2}$ Christopher Charles, ${ }^{2}$ Mark Richards, ${ }^{3}$ Smita Sampath, ${ }^{3}$ Asad Abu Bakar Ali, ${ }^{2}$ Hwa Liang Leo. ${ }^{1}$ Imperial College London, London, UK; ${ }^{2}$ National University of Singapore; ${ }^{3}$ Merck Sharp \& Dohme

10.1136/heartjn-2021-BCS.132

Background Ejection Fraction (EF) has been an important parameter describing cardiac function, because of earlier work demonstrating its correlation with outcomes. However, during conditions such as Heart Failure preserved ejection fraction (HFpEF), EF fails to distinguish HFpEF from healthy patients. There are further reports that EF can be skewed by the geometry of the heart, and Heart Failure (HF) can present a wide variety of cardiac morphologies due to remodelling. The reason for the poor performance of EF and its dependency on geometry is unclear, and it is further unclear if such geometric changes from HF remodelling affects cardiac function. We strive to address this here, and derive an improve and simple EF parameter to resolve this.

Methods We developed a simple discretized numerical model to relate incompressible myocardial strains to stroke volume. We used data from two porcine animal models of heart failure, one for HFpEF and one for HF reduced $\mathrm{EF}$ (HFrEF), and literature clinical measurements to inform our model. We used the model to test the effects of geometric changes relevant to $\mathrm{HF}$ on the ability of the heart to convert myocardial strains to flow function.

Results Our animal models showed that cardiac dilation and wall thickening are primary features relevant to HF. Further investigation via our numerical model showed that wall thickening with no change to strain artificially increased EF, while cardiac dilation with no change to strain artificially decreased $\mathrm{EF}$, demonstrating that $\mathrm{EF}$ can be skewed by geometric 